\title{
Significance of MHD Effects in Stellarator Confinement
}

\author{
A. Weller ${ }^{1}$, S. Sakakibara ${ }^{2}$, K.Y. Watanabe ${ }^{2}$, K. Toi $^{2}$, J. Geiger ${ }^{1}$, M.C. Zarnstorff ${ }^{3}$, \\ S.R. Hudson ${ }^{3}$, A. Reiman ${ }^{3}$, A. Werner ${ }^{1}$, C. Nührenberg ${ }^{1}$, S. Ohdachi ${ }^{2}$, Y. Suzuki ${ }^{2}$, \\ H. Yamada ${ }^{2}$, W7-AS Team ${ }^{1}$, LHD Team ${ }^{2}$
}

${ }^{1}$ Max-Planck-Institut für Plasmaphysik, EURATOM-IPP Association, D-17491 Greifswald, Germany

${ }^{2}$ National Institute for Fusion Science, Toki 509-5292, Japan

${ }^{3}$ Princeton Plasma Physics Laboratory, Princeton, NJ 08543, USA

Corresponding Author:

Dr. Arthur Weller (

Max-Planck-Institut für Plasmaphysik, EURATOM-IPP Association,

Wendelsteinstr. 1, D-17491 Greifswald, Germany

Fax: $+49(0) 3834882509$

Content of Paper:

Main Text: 27 pages (including references and figure captions)

12 Figures, no Table 


\section{Abstract}

Substantial progress has been achieved to raise the plasma beta in stellarators and helical systems by high power neutral beam heating, approaching reactor relevant values [1-3]. The achievement of high- $\beta$ operation is closely linked with configuration effects on the confinement and with magnetohydrodynamic (MHD) stability.

The magnetic configurations of the Wendelstein W7-AS stellarator and of the Large Helical Device (LHD) and their optimization for high- $\beta$ operation within the flexibility of the devices are characterized. A comparative description of the accessible operational regimes in W7-AS and LHD is given. The finite- $\beta$ effects on the flux surfaces depend on the degree of configuration optimization. In particular, a large Shafranov shift is accompanied by formation of islands and stochastic field regions as found by numerical equilibrium studies $[2,4]$. However, the observed pressure gradients indicate some mitigation of the effects on the plasma confinement, presumably because of the high collisionality of high- $\beta$ plasmas and island healing effects (LHD [5-7]). As far as operational limits by pressure driven MHD instabilities are concerned, only weak confinement degradation effects are usually observed, even in linearly unstable regimes.

The impact of the results concerning high- $\beta$ operation in W7-AS and LHD on the future stellarator programme will be discussed, including relations to tokamak research. Some of the future key issues appear to be: - the control of the magnetic configuration (including toroidal current control), - the modification of confinement and MHD properties towards the low collisional regime, - and the compatibility of high- $\beta$ regimes with power and particle exhaust requirements to achieve steady state operation. 


\section{Introduction}

The development of a viable and economic fusion energy source is the basic goal of the international fusion research program. At present, the tokamak is most advanced, and the achievement of a burning plasma state in the International Thermonuclear Experimental Reactor (ITER) [8] will be a crucial milestone in future fusion research based on magnetic confinement. The considerable progress achieved in stellarators and helical sytems appears to provide an even more attractive alternative for a fusion reactor. Stellarators and helical systems have the inherent potential of stable, disruption-free steady state plasma confinement without the necessity for either current drive, control of plasma position and edge flux surface topology, or for active feedback and near-plasma conducting structures to stabilize instabilities. A variety of different magnetic configurations has been realized or proposed. The bumpiness of the non-axisymmetric magnetic field in stellarators determines the confinement and magnetohydrodynamic (MHD) properties which can be optimized by proper threedimensional (3D) shaping of the plasma boundary. "Reverse engineering" has constituted an innovative approach to meet desired physics targets, as realized in the WENDELSTEIN W7-X (Helias concept) [9] and the compact quasi-symmetric stellarator (QS) design (e.g. NCSX, HSX) $[10,11,12]$. On the other hand, configuration optimization within the device flexibility has led to considerably improved plasma confinement in the Large Helical Device (LHD, Heliotron device) without compromising stability significantly [3,13]. This shows, that a sufficient degree of flexibility in present and future stellarator experiments is required in order to quantify tradeoffs between desired properties.

The economic use of fusion power requires volume averaged plasma beta values in the order of $\langle\beta\rangle \approx 5 \%$ (here, beta defined by $\langle\beta\rangle=2 \mu_{0}\left\langle p / B^{2}\right\rangle$, is the volume averaged plasma pressure normalized to the magnetic field pressure). High- $\beta$ operation is closely related to 
MHD equilibrium and stability issues. In this paper, we review the experimental progress of achieving relevant plasma beta in stellarators based on work conducted at WENDELSTEIN W7-AS [1,2] and at the Large Helical Device LHD [3] (section 2). In sub-section 2.1, the two devices and their basic configuration properties are characterized. Section 2.2 describes the optimization of the experimental methods to maximize the achievable plasma beta. Confinement properties and the operational ranges are discussed and compared. A main concern are finite- $\beta$ effects on the magnetic configuration. Section 2.3 deals with equilibrium aspects and associated $\beta$-limits. MHD stability properties and the relevance of MHD instabilities as regards stable high- $\beta$ confinement are discussed in sub-section 2.4 . The final section 3 is devoted to discuss the impact of the results on the future stellarator program and remaining issues.

\section{Progress of High- $\beta$ Operation in Stellarators / Helical Systems}

Before start of LHD operation, beta values in the $1.5-2 \%$ range have been reported by several stellarator experiments including Heliotron-E [14], the Advanced Torsatron Facility ATF [15], the Compact Helical System CHS [16] and W7-AS. Here, we are focusing on more recent results from W7-AS and LHD. A major concern is to compare the results in order to achieve a more comprehensive understanding of the underlying physics of beta induced MHD effects in 3D confinement systems.

\subsection{W7-AS and LHD Configurations}

Wendelstein W7-AS $[17,18]$ is a medium size stellarator $(R=2 \mathrm{~m}, a \leq 0.18 \mathrm{~m})$ operated until mid of 2002. The five-periodic magnetic field is partially optimized in respect of MHD 
properties (reduced Pfirsch-Schlüter currents) and neoclassical transport by 3D shaping. The field $(\mathrm{B} \leq 2.5 \mathrm{~T})$ is generated by a system of 45 nonplanar modular coils providing a low shear rotational transform of $\boldsymbol{t}_{v a c} \approx 0.4$. The vacuum rotational transform can be varied in the range $0.25 \leq \boldsymbol{t}_{\text {vac }} \leq 0.65$ by an extra set of 10 planar toroidal field coils. Additionally, poloidal field coils allow to adjust the horizontal plasma position. Current drive and current control is accomplished by an Ohmic transformer. Since 2000 W7-AS is equipped with a modular island divertor system [19] including a set of 10 in-vessel coils for controlling the width of edge islands by resonant field perturbations $B_{5 m}$. High- $\beta$ plasmas are heated by almost tangential neutral beam injection with beam energies of 50 - $55 \mathrm{keV}$ yielding absorbed heating powers of $P_{N B I} \leq 3.2 \mathrm{MW}$. The heating efficiency decreases towards lower fields restricting high- $\beta$ operation to $\mathrm{B} \geq 0.7 \mathrm{~T}$.

The Large Helical Device (LHD) [20] is the largest existing heliotron type helical device $(R=3.9 \mathrm{~m}, a \leq 0.65 \mathrm{~m})$. The magnetic field of 10 field periods $(\mathrm{B} \leq 3 \mathrm{~T})$ is produced by a pair of superconducting helical windings (heliotron type configuration). Three sets of poloidal field coils are used to change the axis position of the vacuum configuration in the range $R_{a x}=3.4-4.1 \mathrm{~m}$ and for plasma shaping. The profile of the vacuum rotational transform in LHD features much higher shear compared with W7-AS. Central and edge values are in the range $\boldsymbol{t}_{\text {vac }}(0) \geq 0.35$ and $\boldsymbol{t}_{v a c}(a) \leq 1.5$, respectively. The rotational transform can be varied by changing the center of the current in the helical coils. In addition, a set of external saddle coils allows to drive an $\mathrm{n} / \mathrm{m}=1 / 1$ magnetic island at the plasma edge, which can be utilized for local island divertor (LID) operation and island studies [3,7]. The heating power of $P_{N B I} \leq 9.5 \mathrm{MW}$ (absorbed) is provided by 3 tangential beamlines using negative ions with beam energies of $150-180 \mathrm{keV}$. 
The basic configuration parameters of W7-AS and LHD in terms of magnetic shear and magnetic well are compared in figure 1. The profiles were calculated as a function of $\beta$ with the 3D equilibrium code VMEC [21] using model pressure profiles. W7-AS is characterized by low shear allowing to avoid low order iota-resonances, and a magnetic well over the entire plasma (besides at very low $\beta$ due to large inward shift). In LHD, shear is much larger, particularly in the edge region. The inward shifted vacuum configuration $\left(R_{a x}=3.6 \mathrm{~m}\right)$ has no magnetic well, but it develops in the center and expands as $\beta$ is raised. The plasma edge remains in a magnetic well region.

\subsection{High- $\beta$ Operation in W7-AS and LHD: Global Confinement and Operational Range}

The experimental conditions in both devices had to be optimized to develop $\beta$ to the maximum. In W7-AS, several measures and related effects have resulted in quiescent quasistationary discharges with $\beta$ up to $\langle\beta>=3.4 \%$ : firstly, high- $t$ configurations with higher equilibrium limit (see next section) could be exploited. Maximizing of the plasma volume, and hence of the global confinement, was achieved by suppressing the edge islands with the divertor control coils. Secondly, controlling the plasma position in the high- $\beta$ phase and limiting the plasma by the divertor structure allowed to maintain high density H-mode (HDH) confinement [22]. Thirdly, the NBI heating efficiency at low magnetic field could significantly be raised by changing the NBI into an all co-injecting system.

In LHD, a key element is to choose an inward shifted configuration $\left(R_{a x}=3.6 \mathrm{~m}\right)$ which provides significantly enhanced confinement even in the high collisional regime $[13,23]$. Secondly, configurations with minimum Shafranov shift were selected in order to exploit the full NBI power by keeping the plasma center as closely as possible aligned with the radius of tangency of the neutral beams $R_{T, N B I}=3.65-3.7 \mathrm{~m}$. This could be achieved by decreasing the 
coil current pitch parameter $(\gamma=1.254$ 1.22 $)$ resulting in larger aspect ratio $\left(A_{p} \quad R / a \approx 5.8 \quad 6.3\right)$ and higher central rotational transform, and hence in a reduced Shafranov shift. Discharges with $\beta$ up to $<\beta>=4.2 \%$ could be realized.

A summary of the achieved $\beta$ in W7-AS and LHD is shown in figure 2. The W7-AS database contains a selected set of cases, for which an equilibrium analysis with VMEC was available. The LHD database contains an approximate analysis of all shots of the last two experimental campaigns $\left(7^{\text {th }}\right.$ and $\left.8^{\text {th }}\right)$ with $<\beta>>1.5 \%$. A more accurate evaluation requires a more elaborate equilibrium analysis. The diamagnetic $\beta$-values in LHD contain a significant fraction of fast ions; $\langle\beta\rangle_{\text {beam }}$ is estimated to range up to $\sim 1.5 \%$. In both experiments, the flattop time (defined as a time interval for which $\beta$ does not change by more than $10 \%$ ) around the time of analysis ranges up to $\sim 100$ energy confinement times.

The global energy confinement times in both experiments are above the ISS95 scaling [24], as shown in figure 3. In LHD, a progressive degradation of the confinement towards higher $\beta$ is found in the $\mathrm{H}$-factors. This may be caused by a combination of three effects: the increasing violation of MHD stability (see section 2.4), the formation of a stochastic field region (see section 2.3), and a less beneficial confinement scaling at high densities $\left(v^{*}\right)[3,25]$. If the rotational transform (at $\rho \quad r / a=2 / 3$, representing an estimate of the volume average) is formally replaced by an equivalent toroidal current by equating $t_{2 / 3} \cong 1 / q_{95} \propto I_{e q}[1,8,26]$, the confinement can be compared with tokamak scaling laws. Using the mean elongation of the configurations ( $\kappa \approx 2$ for W7-AS, $\kappa \approx 1$ for LHD) yields a reasonable agreement of the W7AS and LHD data with ITER ELMy H-Mode scaling (IPB98(y,2), [8,27]).

The accessible density range differs significantly in W7-AS compared to LHD due to the different heating power densities and magnetic field range. In W7-AS, the plasma volume is 
$\sim 1 \mathrm{~m}^{-3}$ and the maximum $\beta$ is achieved with $\mathrm{B}=0.8 \ldots 0.9 \mathrm{~T}$ compared to $\sim 30 \mathrm{~m}^{-3}$ and $\mathrm{B}=0.45 \ldots 0.5 \mathrm{~T}$ in LHD. Therefore, a much higher density limit (derived from power balance considerations $[28,29]$ ) is predicted for W7-AS. In figure 4, densities of the W7-AS and LHD high- $\beta$ databases are compared with the Sudo limit:

$$
\bar{n}_{\text {Sudo-DL }} \equiv 1.11 \cdot(P \cdot B / V)^{0.5} \quad\left[10^{20} \mathrm{~m}^{-3}\right]
$$

\subsection{Finite- $\beta$ Effects on Equilibrium Topology and Confinement}

The most prominent effect of finite- $\beta$ in toroidal magnetic confinement is the Shafranov shift which leads to a mainly horizontal shift of the magnetic surfaces due to the parallel component of the equilibrium current (Pfirsch-Schlüter current) [30]. In a low- $\beta$, large aspect ratio approximation, the Shafranov shift is

$$
\frac{\Delta}{a} \approx A_{p} \frac{\langle\beta\rangle}{2 t^{2}} \quad\left(A_{p}=R / a\right)
$$

for a classical stellarator, whereas a reduction by $\sim 1 / 2$ is found (as expected) in W7-AS [1]. In LHD, $t \propto A_{p}$ and hence $\Delta / a \propto 1 / A_{p}$. A rough estimate of an equilibrium $\beta$-limit follows from the condition $\Delta / a=1 / 2$ which is considered to lead to a destruction of the equilibrium surfaces. The strong dependence on the rotational transform is reflected in the W7-AS and LHD database analysis as presented in figure 5. The data in both experiments suggest that low rotational transform (which is associated with a lower equilibrium limit) imposes a limit on the $<\beta>$ values that can be achieved.

The beta-induced axis shift has been measured in W7-AS by X-ray tomography [1] and in LHD using Thomson scattering data and and tangential X-ray imaging [31,32]. In LHD, also the shift of the last closed flux surface (LCFS) could be determined. The experimental data are in reasonable agreement with free boundary equilibrium code calculations (VMEC in 
W7-AS, HINT [33] in LHD). The predicted and measured shifts in W7-AS and LHD are compared in figure 6 for typical high- $\beta$ configurations. The LCFS shift in LHD is relatively small due to high edge rotational transform. The normalized shift of the axis relative to the center of the LCFS ( $\Delta / a$, Shafranov shift) is comparable in these particular W7-AS and LHD cases. The slope of $\Delta / a$ is a little more flat in W7-AS, but the absolute value is slightly higher due to an opposite offset of $\Delta / a$ compared to LHD. In both cases, the Shafranov shift remains clearly below the critical value $\Delta / a=1 / 2$.A deeper insight of the equilibrium limit is gained from advanced 3D equilibrium code calculations without the premise of nested flux surfaces. Figure 7 compares the flux surface topology of high- $\beta$ equilibria as obtained from the PIES-code [34] (W7-AS case) and the HINT-code [33] (LHD case). Both, PIES and HINT predict a progressive loss of good flux surfaces with increasing plasma pressure. The significant degree of surface destruction shown in figure 7 corresponds to equilibria close to the maximum achieved $\beta$-values. The cause of the generation of islands and stochastic regions in finite- $\beta$ equilibria are resonant field harmonics of the vacuum field and resonant field perturbations produced by the Pfirsch-Schlüter currents. Therefore, the widths of the perturbations depend on the plasma pressure. The effects could be mitigated to some extent in W7-AS by changing the perturbed field spectrum using the divertor control coils [2]. Surprisingly, the impact of the predicted stochastic field regions on the experimentally observed pressure profiles is only moderate. In both experiments, significant pressure gradients are found in such regions $[2,4,35]$. Therefore, islands and stochastic field regions may not be destructed to the extent predicted by static equilibrium calculations. Actually, healing of an externally driven $\mathrm{m} / \mathrm{n}=1 / 1$ island has been observed in LHD [5-7]. The observation of a critical island size below which the island is annihilated suggests a stabilization mechanism closely related to neoclassical tearing mode (NTM) physics $[7,36]$. 
The expected dependencies of the island size in LHD on $\beta$ and collisionality have been found qualitatively by using an axisymmetric neoclassical bootstrap current model. A proper 3D bootstrap current model is required for a quantitative understanding [7]. Since the plasma in the present high- $\beta$ experiments is in a relatively high collisionality regime (in particular in W7-AS), effects due to an increased ratio of perpendicular to parallel transport may also contribute to reduce the effect of perturbed flux surfaces on the pressure profiles. In any case, indications of enhanced radial transport by parallel conduction in regions of relatively short radial connection lengths are found both in LHD [7] and W7-AS [37].

\subsection{MHD Stability, Impact of MHD Modes on High- $\beta$ Plasmas}

In tokamaks, the operational range of $\beta$ is clearly limited by MHD instabilities such as NTMs, disruptions, resistive wall modes (RWM) or edge localized modes (ELM) when theoretical stability limits are approached. Although the same underlying physics has to be expected, the MHD effects in stellarators seem to be somewhat different. The linear ideal MHD stability properties predicted for W7-AS and LHD high- $\beta$ configurations (as introduced already in section 2.1) are evaluated as a guideline for comparisons with experimental data. A close agreement cannot be expected without using more sophisticated MHD models including non-linear and non-ideal effects. Also, an accurate reconstruction of the equilibrium from experimental data is required to check the agreement with theory, since the stability can sensitively depend on details of the pressure and iota-profiles. A big issue is the treatment of stochastic regions and the determination of the plasma boundary (see end of previous section). A suitable theoretical approach could be to assume averaged flux surfaces in stochastic magnetic field regions [38]. 
In figure 8 , the local ideal interchange stability in terms of Mercier stability diagrams is compared for optimum high- $\beta$ configurations in W7-AS and LHD using fixed model pressure profiles. The configuration of W7-AS is predicted to be unstable below $\langle\beta\rangle=1.5 \%$ across the whole plasma radius due to the flat shear and the transition from magnetic hill in the vacuum configuration to a magnetic well at finite $\beta$ (see figure 1a). The edge remains unstable because the pressure gradient rises towards the plasma boundary (available equilibrium reconstructions were made with parabolic pressure profiles). Low order rational surfaces at $\boldsymbol{t}=1 / 2$ and $\boldsymbol{t}=1 / 3$ appear during the formation of a broad stable region along with increased shear, moving inward as pressure and shear is further increased. The unstable region in the LHD configuration with axis position $R_{a x}=3.75 \mathrm{~m}$ is rather small because of a marginal magnetic hill region in the center and effective shear stabilization. However, as already mentioned in section 2.2, the confinement is unfavourable. The inward shifted configuration with $R_{a x}=3.6 \mathrm{~m}$ has good confinement properties, but the unstable region is much wider in radius and extended over a broader range in $\beta$ because of an increased magnetic hill region (the dashed lines indicate the separation between magnetic hill and magnetic well regions) [39].

The path towards high- $\beta$ plasmas has to traverse regions predicted to be unstable with respect to ideal interchange modes and global (low-n) ideal modes. The violation of the Mercier stability criterion, however, does not inhibit the access to higher $\beta$ in both experiments by using higher heating power. Global low-n interchange modes are found in W7-AS [1,2,40] and LHD [41-45], mostly consistent with predictions based on the linear MHD stability theory. However, in most cases the observed modes saturate on a harmless level. Therefore, the low-n linear stability threshold significantly underestimates the achievable $\beta$. The typical behaviour of high- $\beta$ discharges in W7-AS with respect to MHD 
modes is shown in figure 9. The range of $\beta$ where the pressure driven $\mathrm{m}=2$ global mode is seen by magnetic diagnostics and X-ray tomography is consistent with predictions by the ideal global mode analysis with the CAS3D code [46,47]. At the maximum $\beta$, the discharges are very quiescent consistent with linear stability prediction for low-n and high-n ideal ballooning modes.

Extended MHD stability studies have been performed in LHD. In particular, experimentally determined $\beta$-gradients are compared with linear stability thresholds and with observed mode amplitudes [31,35,48,49]. Figure 10 contains an local analysis for a surface close to the plasma perphery and for a surface in the plasma core. In spite of mode activity in the Mercier unstable region, the $\beta$-gradients can be increased. However, unstable low-n interchange modes with growth rates $\gamma / \omega_{A} \geq 10^{-2} \quad\left(\omega_{A}=v_{A} / R\right)$, corresponding to a radial width of the modes of $\delta / a \sim 5 \%$, seem to be relevant and limit the achievable $\beta$-gradients in the unstable region. In the stability analysis effects due to bootstrap and Ohkawa currents $(\leq 25 \mathrm{kA} / \mathrm{T})$ were neglected. This is a reasonable assumption at the plasma edge (Fig. 10, left), since the increment of iota due to the currents is less than 0.03 . However, in the plasma core (Fig. 10, right) the currentless assumption corresponds to an upper stability limit, since the current in the selected co- and balanced NBI cases reduces the ideal interchange stability via the change of the equilibrium [50]. The low-n ideal MHD unstable boundaries are derived from calculations with the 3D MHD stability code TERPSICHORE [51] using various beta values and pressure profiles [49]. High-n ideal ballooning modes are expected to have very similar stability thresholds. Core MHD is effectively stabilized by the pressure induced formation and progressive deepening of a magnetic well. This self-stabilization mechanism is similar as in W7-AS. In contrast, the ideal stability at the plasma edge relies entirely on magnetic shear. The amplitudes of observed edge MHD modes typically increase with rising 
$\beta$. Therefore, the edge stability is crucial for maintaining broad pressure profiles with large edge gradients, in particular, if shear stabilization is annhilated by resistive effects.

The favourable LHD configurations for achieving maximum $\beta$ (reduced $\gamma$-parameter, increased aspect ratio and central rotational transform, $R_{a x}=3.6 \mathrm{~m}$ ) are more unstable because of lower shear and restricted magnetic well formation (reduced Shafranov shift). The $t=1$ surface is located further inward, in particular when a significant plasma current (mostly Ohkawa current) increases the external current rotational transform. In this case the ideal $\mathrm{m} / \mathrm{n}=1 / 1$ is unstable at low- $\beta$, and strong activity including internal disruptions in the core region are found. An example of a large MHD induced collapse is presented in figure 11. Internal disruptions are also seen at other rational surfaces $(t=1 / 2,1 / 3$, etc. $)$ if present in the plasma core. But the effect on the confinement is much larger in the case with $t=1$ [52].

Indications of resistive MHD effects have been found in W7-AS and LHD. The magnetic Reynolds number $S=\tau_{\text {res }} / \tau_{A}$ for high- $\beta$ conditions is typical $\mathrm{S} \sim 10^{5}$ in W7-AS and $\mathrm{S} \sim 10^{6}$ in LHD. Fast thermal collapses occur in W7-AS high- $\beta$ cases in correlation with decreased electron temperature and low rotational transform [53]. The fast collapse of the plasma energy $(\sim 100 \mu \mathrm{s})$ is accompanied by a magnetic spike of same duration. The observed scaling of this instability with plasma and configuration parameters is consistent with that of resistive ballooning modes, if linear growth rates [54] are used as a measure of the expected mode activity: $\gamma \approx\left(\langle\beta\rangle /\langle\beta\rangle_{\mathrm{c}}\right) \eta k^{2} / \mu_{0} \propto\left(\langle\beta\rangle / t^{2}\right) T_{e}^{-3 / 2}$, where $\langle\beta\rangle_{\mathrm{c}} \propto t^{2}$ is the ideal ballooning limit which roughly coincides with the equilibrium $\beta$-limit. The linear dependence of $\gamma$ on $\eta$ results from the so called resistive pressure convection limit model with the additional conditions of low growth rates and sufficiently low resistivity [55,56,57]. Only qualitative considerations have been made about the validity of these conditions. Since the resistivity of 
high-beta plasmas in LHD is much lower than in W7-AS, the stabilising effect by plasma compression is more important in LHD and the $\gamma \sim \eta^{1 / 3}$ regime can then be recovered. Figure 12 shows a W7-AS dataset containing high- $\beta$ database including all cases where fast collapses were observed together with stable cases. In the diagram, $\left\langle\beta>\right.$ normalised to $t^{2}$ is plotted versus $T_{e}^{3 / 2}$. Almost all data are bounded by the dashed line representing an empirical value for a critical "stability parameter" defined by the inverse linear growth rate $s \propto \gamma^{-1}$.

The edge MHD modes in LHD exhibit clearly features of resistive interchange modes. The increase of the edge mode amplitudes up to the maximum $\beta$ is not consistent with ideal mode stability and reflects the reduction of shear stabilization towards high $\beta$ (correlated with low S). Actually, the scaling of the mode amplitudes is consistent with that predicted for linear growth rates of resistive interchange modes according to $\gamma \propto S^{-1 / 3}$ [58]. This has been found in detailed studies in LHD [4,59] including a comparison with CHS results [44]. It may be concluded, that resistive interchange or ballooning modes will not be relevant for reactor grade plasmas, in particular, if advanced physics models including two-fluid theory are taken into account [60].

\section{Discussion and Conclusions}

The results obtained in the high- $\beta$ regime in W7-AS and LHD are considered to to be of great relevance for evaluating the prospects of the future stellarator program. The two new devices presently under construction, W7-X and NCSX, are both extensively optimized with respect to confinement and MHD properties. But they are located in totally different corners of the configuration space, and this will provide another wealth of new results.

The configuration and size of the W7-AS and LHD devices is rather different, too. However, the global confinement is comparable as follows from the comparison with 
common scaling laws. In order to achieve good confinement, the LHD configuration has to be optimized by exploiting the flexibility of the device, but implicating reduced stability. However, deleterious effects of MHD instabilities on the confinement in LHD have not been found so far or can be avoided, even in unstable regimes. Therefore, one might argue that configuration optimization, as partially achieved in W7-AS or more completely implemented in W7-X, may not be required to such an extent. In Helias systems (W7-X), however, a tradeoff between different desired configuration properties is not necessary. The most important goals including good confinement, good equilibrium surfaces and sufficient high MHD stability can be achieved simultaneously.

The successful test of the island divertor concept in W7-AS and the demonstration of steady state plasma operation in LHD using the local island divertor head are important milestones towards power handling and exhaust control required for steady state systems. Long pulse experiments in LHD with moderate heating power have resulted in a pulse of $1905 \mathrm{~s}$ duration and a total input heating energy of $1.3 \mathrm{GJ}$ exceeding the previous record by Tore Supra of 1.07 GJ. W7-X is designed to sustain heating powers up to $10 \mathrm{MW}$ for $30 \mathrm{~min}$. Demonstration of this capability will be another important step.

A crucial issue for each stellarator and helical device is to establish plasma core conditions with good confinement (but without impurity accumulation) compatible with steady state operation. The HDH mode in W7-AS [22] is a favoured scenario compliant with high- $\beta$ parameters $[1,2]$. However, it remains a big issue whether this regime can be attained in W7-X, since the density threshold in W7-AS is larger than the achievable densities in W7-X according to density limit scaling laws [28,29]. In this respect, the expectations concerning the achievable plasma $\beta$ and density are close to those in LHD. 
In the high- $\beta$ regime, a convincing demonstration of steady state power handling is still missing. The biggest issue connected to this seems to be keeping control of the edge topology as $\beta$ is increased. In the present devices, the relative large Shafranov shift, the progressive stochastization of the outer plasma region and the change of the configuration by net toroidal current such as bootstrap and Ohkawa currents are not compatible with proper island divertor operation. External current drive by electron cyclotron waves may be a possibility to maintain current-free operation in future large devices $[61,62]$.

With respect to the Shafranov shift, the studies in W7-AS and LHD, but also in CHS [16], have shown a good agreement with 3D equilibrium code calculations with VMEC, HINT and PIES. In particular, the partial optimization of the W7-AS configuration resulting in reduced Shafranov shift could be verified [1]. Therefore, the expected equilibrium properties for W7-X (and NCSX) seem to be reliable given the available equilibrium benchmarking. In particular, the stiffness of the W7-X configuration should ensure to maintain a sufficiently fixed profile of the rotational transform and hence a passively stable island divertor configuration.

As regards the pressure induced destruction of magnetic surfaces, the predictions by PIES and HINT have provided more insight in the physics of the equilibrium limit. But also, the analyses have posed new issues as to which extent plasma dynamics has to be included in the equilibrium models to make allowance for island healing effects. In connection with it, the equilibrium reconstruction in the presence of finite pressure gradients in an ergodic edge region requires additional efforts. Nevertheless, the PIES calculations for W7-X [63] and NCSX [11] give much better results in the high- $\beta$ regime. This is achieved in W7-X by the significant reduction of the Pfirsch-Schlüter current and the optimized coil field spectrum. In NCSX, a particular "coil healing" procedure is applied to compensate contributions due to 
equilibrium currents by those of resonant coil perturbations $[64,65]$. Therefore, a sufficiently high quality of finite- $\beta$ flux surfaces may be expected in W7-X and in NCSX as well.

Also, the effect of net currents on the edge topology is considered to pose no big problem in W7-X. An important goal was to eliminate the bootstrap current along with the reduction of the Pfirsch-Schlüter current in order to keep passive control of the magnetic configuration. This is of course quite different than in NCSX, where bootstrap current drive is an essential element of the QAS approach. The generation and control of substantial plasma current and the maintenance of kink stability in NCSX imposes greater shape control requirements than in previous stellarators. NCSX is a proof-of-principle experiment which has to demonstrate the novel physical concept and its technical realization The challenge for the future will be to incorporate the features required for reactor capability, i.e. to develop a compatible power exhaust system and to minimize active configuration control.

Concerning MHD stability an important result consists in the absence of fast disruptive instabilities even close to operational limits determined by equilibrium deterioration, density limit or confinement (power). The plasma reaction mostly occurs via slow transitions to increased transport. This is an important advantage compared with tokamak devices where disruptions usually inhibit the approach or exceeding of operational limits. Although the local ideal MHD stability seems to underestimate the stability found in W7-AS and LHD experiments, the linearized ideal MHD analyses are still useful. The observed mode activity, even if not always relevant for the confinement, is mostly correlated with the predicted unstable regions. An important nonlinear effect could be mode saturation on a low level due to local pressure profile flattening [66]. The data suggest that relevant MHD modes are absent, if Mercier stability is ensured. Another important finding at LHD is the identification of "confinement relevant" modes correlated with ideal low-n interchange modes of a 
sufficiently large growth rate and radial width ( $\geq 5 \%$ of plasma radius). These modes provide a good estimate of an ideal stability limit which cannot be exceeded in LHD. A stability index based on a critical width of MHD modes may provide a useful criterion to assess relevant MHD limits and to optimise stellarator configurations.

Given these results, the prospects for MHD stability in W7-X are very promising allowing to achieve stable high- $\beta$ plasma operation. NCSX has to face more efforts to achieve a stationary current profile and to control the iota-profile and plasma shape during the path to high- $\beta$. The LHD device has reached the record of $\langle\beta\rangle \geq 4 \%$ in stellarators and helical devices without being limited by deleterious MHD instabilities. However, the requirements with respect to equilibrium (low Shafranov shift) and stability (sufficient magnetic well depth), as well as with respect to confinement (inward shifted configuration favourable) and stability (outward shift favourable) are conflicting. Also, the magnetic hill region with large magnetic shear may be susceptible not only to resistive but also to ideal interchange modes at higher $\beta$ and to field stochastization. More detailed studies of the stability limit in LHD seem to be feasible if higher heating power is available.

Another remaining issue in the future stellarator program, not discussed so far, are Alfvén and fishbone instabilities excited by resonances with energetic particles. Besides the modes well known from tokamak research stellarator Alfvén eigenmodes (AE) such as helicity induced AEs (HAE) or mirror induced AEs (MAE) have been predicted $[47,67,68]$ and found in the W7-AS and LHD experiments $[1,40,42,69]$. Usually, they do not play an important role under the present high- $\beta$ conditions because the fraction of resonant fast injected ions is relatively small. However, Alfvén modes may be of great relevance in reactor-like plasmas.

It seems to be very important in future experimental devices to provide sufficient flexibility for changing and controlling the magnetic configuration. This will be realized to a 
large extent in W7-X and NCSX. External current drive capability should be available depending on the magnitude of the bootstrap and NBI currents appearing in experiment. Also, advanced diagnostic systems are required to get reliable reconstructions of the equilibrium profiles. Together with the development of more sophisticated physics MHD models this will allow one to prepare a good physics basis for a stellarator fusion reactor.

\section{Acknowledgement}

The first author (A. W.) would like to acknowledge his invitation by NIFS as Foreign Research Staff (Guest Professor). 


\section{References}

[1] A. Weller et al, "Experiments close to the beta-limit in W7-AS", Plasma Phys. Control. Fusion 45 (2003) A285-A308.

[2] M.C. Zarnstorff et al, "Equilibrium and Stability of High-Beta Plasmas in Wendelstein 7-AS", $21^{\text {th }}$ IAEA Fusion Energy Conference, 1-6 November 2004, Vilamoura, Portugal, EX/3-4.

[3] O. Motojima et al, "Confinement and MHD stability in the Large Helical Device", $21^{\text {th }}$ IAEA Fusion Energy Conference, 1-6 November 2004, Vilamoura, Portugal, EX/3-4., OV1-4.

[4] K.Y. Watanabe, S. Sakakibara, "Subjects on MHD equilibrium in the LHD experiments", US-Japan JIFT Workshop, 25-27 January 2005, Kyoto, Japan.

[5] Y. Nagayama et al, "Experiment of magnetic island formation in Large Helical Device", Nucl. Fusion 45 (2005) 888-893.

[6] R. Kanno et al, "Formation and healing of $n=1$ magnetic islands in LHD equilibrium", Nucl. Fusion 45 (2005) 888-893.

[7] N. Ohyabu et al, "Bifurcation of equilibria between with and without a large island in the large helical device”, Plasma Phys. Control. Fusion 47 (2005) 1431-1437.

[8] ITER Teams, Nucl. Fusion 36 (1999) 2137-2638.

[9] J. Nührenberg and R. Zille, "Stable stellarators with medium $\beta$ and aspect ratio“, Phys. Lett. A 114 (1986) 129.

[10] S.P. Hirshman et al, "Physics of compact stellarators", Physics of Plasmas 6 (1999) 1858.

[11] M.C. Zarnstorff et al, "Physics of the compact advanced stellarator NCSX", Plasma Phys. Control. Fusion 43 (2001) A237-A249.

[12] F.S.B. Anderson et al, "The helically symmetric experiment (HSX) goals, design and status", Fusion Technol. 27 (1995) 273.

[13] H. Yamada et al, "Configuration flexibility and extended regimes in Large Helical Device", Plasma Phys. Control. Fusion 43 (2001) A55-A71.

[14] M. Wakatani and S. Sudo, "Overview of Heliotron-E results", Plasma Phys. Control. Fusion 38 (1996) 933-988. 
[15] J.H. Harris et al, "Second Stability in the ATF Torsatron", Physical Review Letters 63 (1989) 1249-1252.

[16] S. Okamura et al, "High beta discharges with neutral beam injection in CHS", Nucl. Fusion 35 (1995) 283-296.

[17] H. Renner et al, "Initial operation of the Wendelstein 7AS advanced stellarator", Plasma Physics and Controlled Fusion 31 (1989) 1579-1596.

[18] F. Wagner et al, "W7-AS: One step of the Wendelstein stellarator line", Physics of Plasmas 12 (2005) 072509.

[19] P. Grigull et al, "First island divertor experiments on the W7-AS stellarator", Plasma Phys. Control. Fusion 43 (2001) A175-A193.

[20] A. Iiyoshi et al, "Overview of the Large Helical Device project", Nucl. Fusion 39 (1999) 1245.

[21] S.P. Hirshman et al, "Improved radial differencing for three-dimensional magnetohydrodynamic equilibrium calculations", J. Comp. Phys. 87 (1990) 396.

[22] K. McCormick et al, "New Advanced Operational Regime on the W7-AS Stellarator", Phys. Rev. Lett. 89 (2002) 015001-1

[23] O. Motojima et al, "Recent advances in the LHD experiment", Nucl. Fusion 43 (2003) 1674-1683.

[24] U. Stroth et al, "Energy confinement scaling from the international stellarator database", Nucl. Fusion 36 (1996) 1063.

[25] J. Miyazawa et al, "Temperature dependence of the thermal diffusivity in high-collisionality regimes in the large helical device”, Plasma Phys. Control. Fusion 47 (2005) 801-813.

[26] N.A. Uckan, “ITER Physics Design Guidelines”, IAEA, Vienna (1990) 10.

[27] H. Yamada et al, "Characterization of edge pressure in the Large Helical Device", Plasma Phys. Control. Fusion 44 (2002) A245-A251.

[28] S. Sudo et al, "Scalings of energy confinement and density limit in Stellarator/Heliotron devices", Nucl. Fusion 30 (1990) 11.

[29] L. Giannone et al, "Radiation power profiles and density limit with a divertor in the W7-AS stellarator", Plasma Phys. Control. Fusion 44 (2002) 2149-2165.

[30] H. Wobig, "The theoretical basis of a drift-optimized stellarator reactor", Plasma Physics and Controlled Fusion 35 (1993) 903. 
[31] K.Y. Watanabe et al, "Progress of High-Beta Experiments in Stellarator/Heliotron", Fusion Science \&Technology 46 (2004) 24-33.

[32] T. Kobuchi et al, "Shafranov Shift Measurement Using Soft X-ray CCD Camera on Large Helical Device", $31^{\text {st }}$ EPS (London, UK) ECA Vol. 28B (2004) P-5.115.

[33] K. Harafuji K, T. Hayashi and T. Sato, "Computational study of three-dimensional magnetohydrodynamic equilibria in toroidal helical systems”, J. Comput. Phys. 81 (1989) 169.

[34] A.H. Reiman and H. Greenside, "Numerical solution of three-dimensional magnetic differential equations", J. Comput. Phys. 75 (1988) 423.

[35] K.Y. Watanabe, "Effects of configuration on transport and MHD phenomena", MHD Meeting on Magnetic Field Structure and Improvement of MHD Stability by Pressure Profile Control, 18 February 2005, NIFS, Toki-shi, Japan.

[36] K. Itoh, S.-I. Itoh and M. Yagi, "Self-sustained annihilation of magnetic islands in helical plasmas", Physics of Plasmas 12 (2005) 072512.

[37] M.C. Zarnstorff et al, "The Saturation of Beta in W7-AS", $32^{\text {nd }}$ EPS Plasma Physics Conference, 27 June - 1 July 2005, Tarragona, Spain, Europhysics Conference Abstracts (ECA) Volume 29C, P-1.062.

[38] N. Nakajima et al, "Boundary modulation effects on MHD instabilities in Heliotrons", $21^{\text {th }}$ IAEA Fusion Energy Conference, 1-6 November 2004, Vilamoura, Portugal, TH5-6.

[39] M. Fujiwara et al, “Overview of LHD experiments”, Nucl. Fusion 41 (2001) 1355-1367.

[40] A. Weller et al, "Survey of magnetohydrodynamic instabilities in the advanced stellarator Wendelstein 7-AS”, Physics of Plasmas 9 (2001) 931-956.

[41] S. Sakakibara et al, "Effect of MHD activities on pressure profile in high- $\beta$ plasmas of LHD", Plasma Phys. Control. Fusion 44 (2002) A217-223.

[42] K. Toi et al, "MHD instabilities and their effects on plasma confinement in Large Helical Device plasmas", Nucl. Fusion 44 (2004) 217-225.

[43] S. Sakakibara et al, "MHD Activities in high- regime of LHD”, $31^{\text {st }}$ EPS (London, UK) ECA Vol. 28G (2004) O-4.01.

[44] S. Sakakibara et al, "MHD characteristics in the high beta regime of the Large Helical Device", Nucl. Fusion 41 (2001) 1177-1183. .

[45] K.Y. Watanabe et al, "Effects of global MHD instability on operational high beta-regime in LHD”, Nucl. Fusion 45 (2005) 1247-1254. 
[46] C. Schwab, "Ideal magnetohydrodynamics: Global mode analysis of three-dimensional plasma configurations", Phys. Fluids B5 (1993) 3195.

[47] C. Nührenberg, "Compressional ideal magnetohydrodynamics: Unstable global modes, stable spectra, and Alfvén eigenmodes in Wendelstein 7-X-type equilibria”, Physics of Plasmas 6 (1999) 137-147.

[48] A. Komori et al, "Recent results from the Large Helical Device“, Plasma Phys. Control. Fusion 45 (2003) 671-686.

[49] K.Y. Watanabe et al, "Relationships between the prediction of Linear MHD Stability Criteria and the experiment in LHD“, J. Plasma Fusion Res. SERIES, 6 (2004) 523-526.

[50] K. Ichiguchi et al, "Effects of net toroidal current profile on the Mercier criterion in heliotron plasmas", Nucl. Fusion 42 (2002) 557-567.

[51] W.A. Cooper, "Variational Formulation of the linear MHD Stability os 3D Plasmas with noninteracting hot Electrons“, Plasma Phys. Control. Fusion 34 (1992) 1011-1036.

[52] S. Ohdachi, "Internal disruptions observed by the high speed tangentially viewing soft X-ray camera", MHD Meeting on Magnetic Field Structure and Improvement of MHD Stability by Pressure Profile Control, 18 Februaray 2005, NIFS, Toki-shi, Japan.

[53] A. Weller et al, "MHD Effects related to High-Beta Operation in WENDELSTEIN W7-AS", $32^{\text {nd }}$ EPS Plasma Physics Conference, 27 June - 1 July 2005, Tarragona, Spain, Europhysics Conference Abstracts (ECA) Volume 29C, P-4.056.

[54] K. Miyamoto, "Plasma Physics for Nuclear Fusion”, MIT Press, 1980.

[55] G. Bateman and D.B. Nelson, "Resistive-Ballooning-Mode Equation", Phys. Rev. Lett. 41 (1978) 1805-1807. .

[56] R. Sánchez et al, "Compressibility Effects on Ideal and Resistive Ballooning Stability in the TJ-II Heliac Device", Nucl. Fusion 37 (1997) 1363-1373.

[57] R. Kaiser, "Resistive Ballooning Modes in W7-AS and W7-X" Nucl. Fusion 33 (1993) 1281-1291.

[58] K. Ichiguchi et al, "Ideal and resistive pressure gradient driven instabilities in Heliotron DR", Nucl. Fusion 29 (1989) 2093.

[59] S. Sakakibara et al, "Recent Progress of MHD Study in High- $\beta$ Plasmas of LHD", $15^{\text {th }}$ Intern. Stellarator Workshop, 3-7 October 2005, Madrid, Spain, IT-07.

[60] H.R. Strauss et al, "Simulation of two fluid and energetic particle effects in stellarators", 
Nucl. Fusion 44 (2004) 1008-1014.

[61] Yu. Turkin et al, "Current control by ECCD for W7-X", $15^{\text {th }}$ Intern. Stellarator Workshop, 3-7 October 2005, Madrid, Spain, OT-10.

[62] S. Ferrando i Margalet et al, "Electron Cyclotron Current Drive Compensation of the Bootstrap Current in Quasi-symmetric Reactor Devices", $15^{\text {th }}$ Intern. Stellarator Workshop, 3-7 October 2005, Madrid, Spain, P1-27.

[63] M. Drevlak et al, "PIES free boundary stellarator equilibria with improved initial conditions", Nucl. Fusion 45 (2005) 731-740.

[64] S.R. Hudson et al, "Free-boundary full-pressure island healing in stellarator equilibria: coil-healing”, Plasma Phys. Control. Fusion 44 (2002) 1377-1382.

[65] S.R. Hudson et al, "Constructing integrable high-pressure full-current free-boundary stellarator magnetohydrodynamic equilibrium solutions", Nucl. Fusion 43 (2003) 1040-1046.

[66] K. Ichiguchi et al, "Nonlinear MHD analysis for LHD plasmas", Nucl. Fusion 43 (2003) 1101-1109.

[67] N. Nakajima et al, "High- $n$ helicity-induced shear Alfvén eigenmodes", Phys. Fluids B4 (1992) 1115.

[68] Ya. I. Kolesnichenko et al, "Alfvén continuum and high-frequency eigenmodes in optimized stellarators", Physics of Plasmas 8 (2001) 491-509.

[69] K. Toi et al, "Energetic ion driven Alfvén eigenmodes in Large Helical Device plasmas with three-dimensional magnetic structure and their impact on energetic ion transport", Plasma Phys. Control. Fusion 46 (2004) S1-S13. 


\section{Figure Captions}

Figure 1. Finite- $\beta$ effects on radial profiles of the rotational transform $t$ and the magnetic well depth $V^{\prime \prime}(\rho) / V^{\prime}(0)(V$ :volume, $\rho=r / a)$ calculated by the VMEC code for W7-AS (left) and LHD (right). The vacuum configurations are inward shifted ( $R_{a x}=3.6 \mathrm{~m}$ in LHD).

Figure 2. High- $\beta$ databases of W7-AS (left) and LHD (right, $7^{\text {th }} \& 8^{\text {th }}$ campaign). Volume averaged $\beta$ values are given as a function of the flattop time normalized to the confinement time. The open symbols refer to the time in the discharge, where the VMEC analysis was performed. The solid symbols represent time averages during the flattop. In addition, the typical range of tokamak data is indicated by the shaded area.

Figure 3. Comparison of experimental energy confinement times with the ISS95 scaling for same W7-AS and LHD high- $\beta$ databases as used in fig. 2 (left). The H-factors (enhancement $\left.\tau_{E, \text { exp }} / \tau_{E, I S S 95}\right)$ are given on the right.

Figure 4. Densities for same W7-AS and LHD high- $\beta$ databases as used in fig. 2 (left) compared with Sudo density limit [28] (dashed line).

Figure 5. Achieved $\left\langle\beta>\right.$ in W7-AS and LHD plotted versus $c t^{2} / A_{p} \equiv\langle\beta\rangle_{\text {eql }}$ as an estimate of an "equilibrium $\beta$ limit" corresponding to $\Delta / a=1 / 2 \quad(\mathrm{c}=2$ for W7-AS, $\mathrm{c}=1$ for LHD). The solid line represents $\langle\beta\rangle=\langle\beta\rangle_{\text {eql }}$ 
Figure 6. Free boundary VMEC (W7-AS, left) and HINT (LHD, right) predictions of $\beta$ induced horizontal shifts of the plasma axis (red), the LCFS (blue) and their difference (Shafranov shift normalized to horizontal plasma radius $a_{h}$, black dashed line, right scales). The shaded areas represent the range of available experimental data.

Figure 7. Free boundary high- $\beta$ equilibria as calculated for W7-AS with PIES (left) and for LHD with HINT (right). A large fraction of flux surfaces is predicted to be degraded by island formation and generation of stochastic field regions.

Figure 8. Mercier stability diagrams for W7-AS (left) and LHD (right) configurations. The W7-AS and LHD $\left(R_{a x}=3.6 \mathrm{~m}\right)$ configurations are inward shifted and less stable.

Figure 9. Mode amplitudes in W7-AS depending on $\beta$. The observed $\mathrm{m} / \mathrm{n}=2 / 1$ pressure driven mode (left, inset: X-ray tomogram) dissappears above $<\beta>=2.5 \%$ in agreement with vanishing growth rates of global ideal modes as predicted by CAS3D (right).

Figure 10. Observed gradients of $\beta$ in LHD at the plasma periphery ( $\rho=0.9$, left) and in the core $(\rho=0.5$, right) plotted against $\langle\beta\rangle$. Stability thresholds for the Mercier stability are significantly violated in experiment. The threshold for low-n ideal interchange modes with growth rates $\gamma / \omega_{A}=10^{-2}$ is not exceeded in experiment. The presence of observed MHD modes correlates with the Mercier unstable region. 
Figure 11. Collapse of plasma energy in LHD due to an $\mathrm{m} / \mathrm{n}=1 / 1$ interchange mode. A small $\gamma$-parameter $(\gamma=1.129)$ along with co-current generates a low shear iota-profile with $t=1$ in the plasma core. A flattened region in the $T_{e}$-profile is formed during the collapse (right).

Figure 12. Database of $\left\langle\beta>\right.$ normalized to $t^{2}$ versus $T_{e}^{3 / 2}$ of high- $\beta$ discharges in W7-AS. The parameters just prior to MHD collapses (solid triangles) are close to a critical value of the inverse linear growth rate (dotted line "s $=0.5$ "). The solid lines are the trajectories of individual discharges until the collapse. 


\section{Figures}
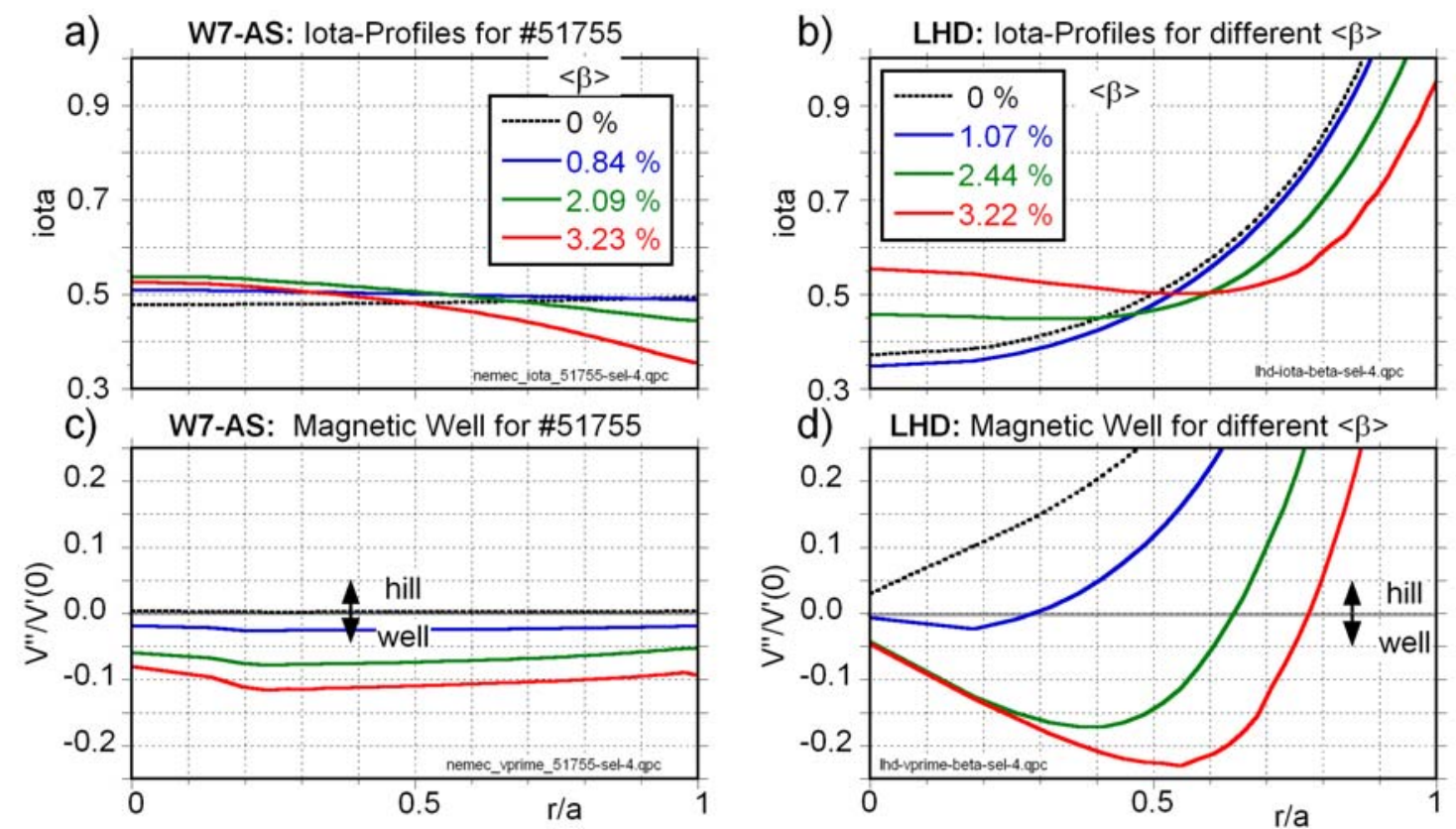

Figure 1. Finite- $\beta$ effects on radial profiles of the rotational transform $t$ and the magnetic well depth $V^{\prime \prime}(\rho) / V^{\prime}(0)(V$ :volume, $\rho=r / a)$ calculated by the VMEC code for W7-AS (left) and LHD (right). The vacuum configurations are inward shifted ( $R_{a x}=3.6 \mathrm{~m}$ in LHD). 

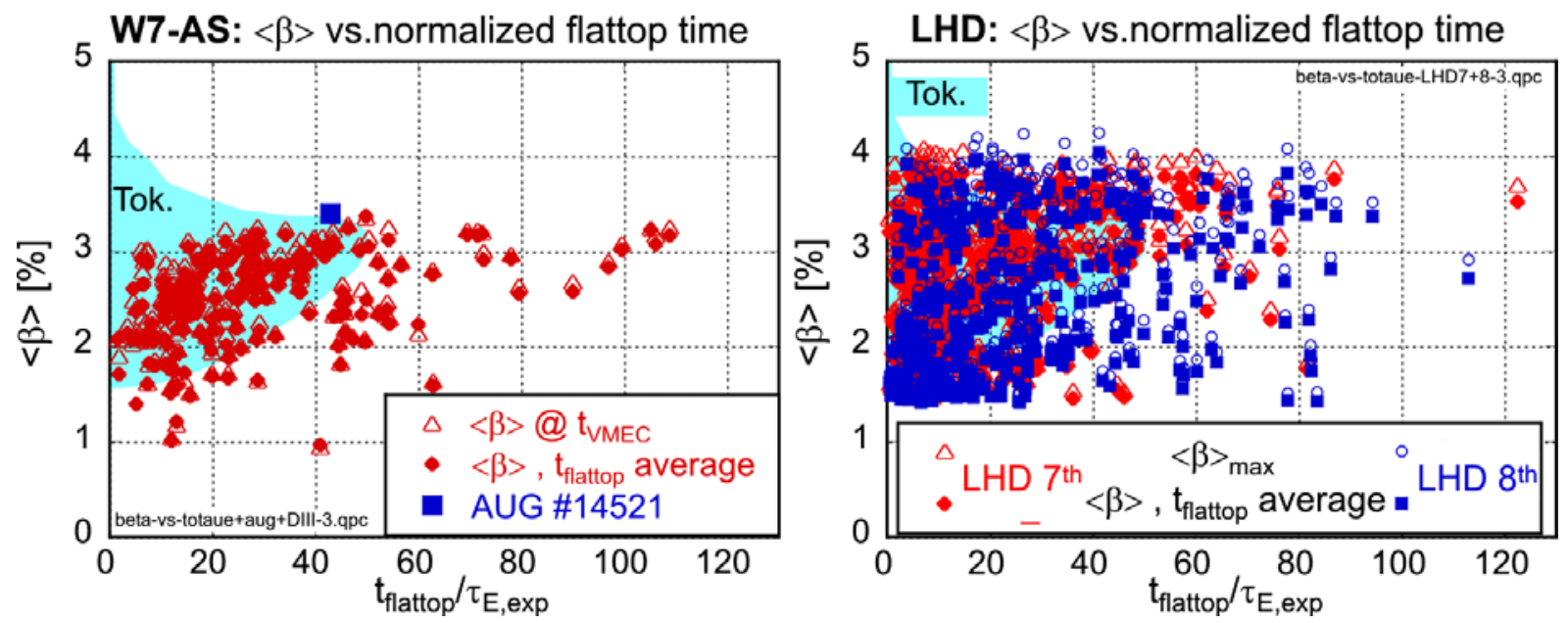

Figure 2. High- $\beta$ databases of W7-AS (left) and LHD (right, $7^{\text {th }} \& 8^{\text {th }}$ campaign). Volume averaged $\beta$ values are given as a function of the flattop time normalized to the confinement time. The open symbols refer to the time in the discharge, where the VMEC analysis was performed. The solid symbols represent time averages during the flattop. In addition, the typical range of tokamak data is indicated by the shaded area. 

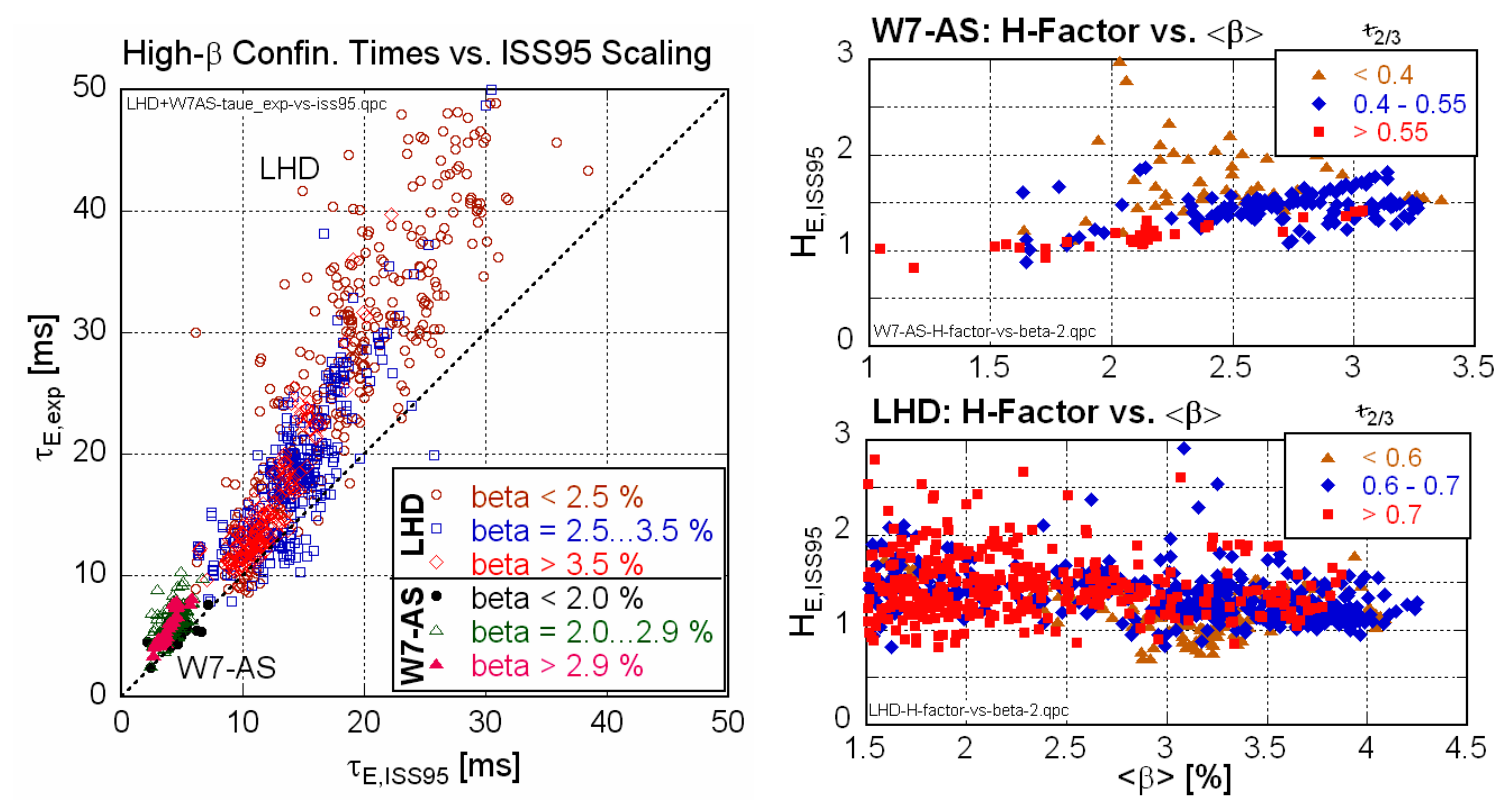

Figure 3. Comparison of experimental energy confinement times with the ISS95 scaling for same W7-AS and LHD high- $\beta$ databases as used in fig. 2 (left). The H-factors (enhancement $\left.\tau_{E, \exp } / \tau_{E, I S S 95}\right)$ are given on the right. 


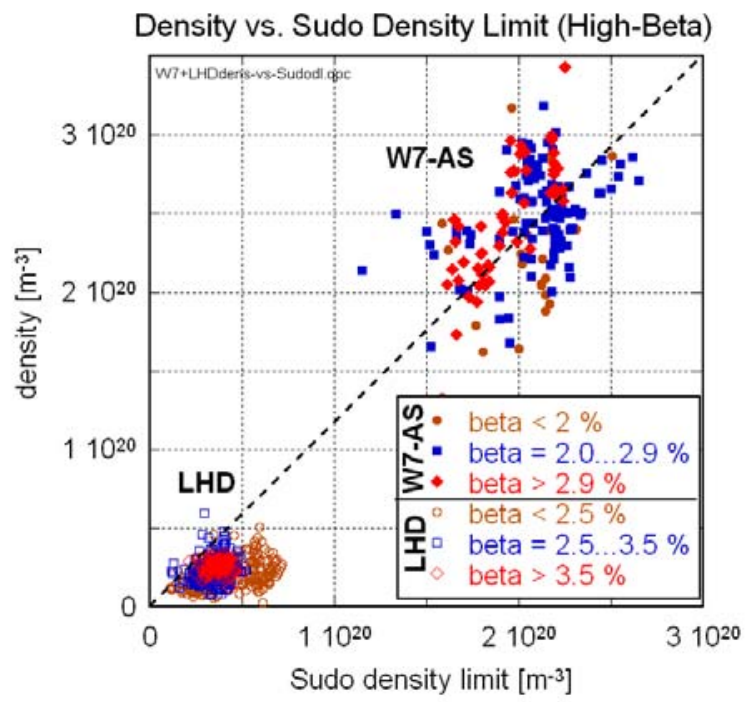

Figure 4. Densities for same W7-AS and LHD high- $\beta$ databases as used in fig. 2 (left) compared with Sudo density limit [28] (dashed line). 

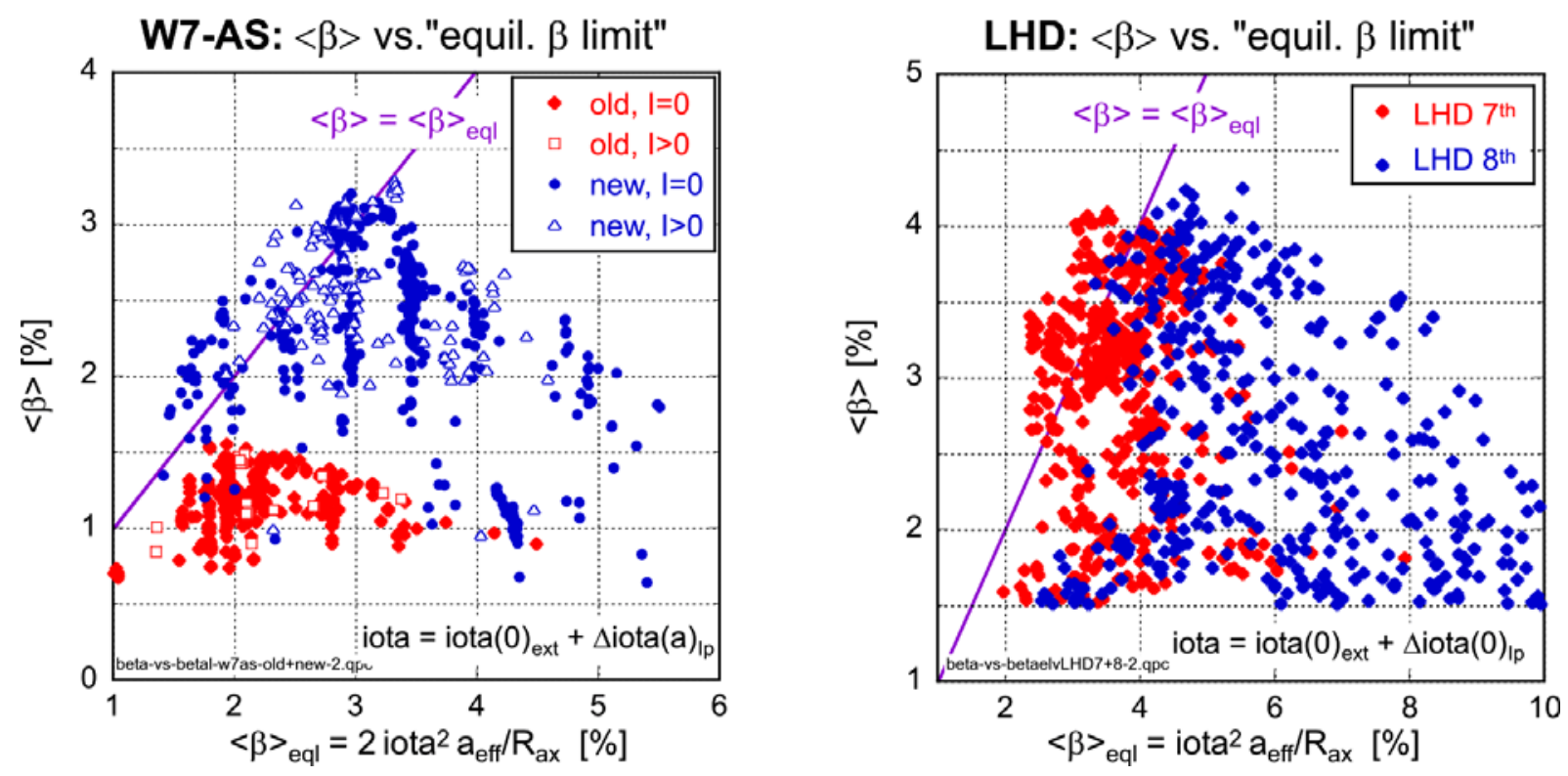

Figure 5. Achieved $\left\langle\beta>\right.$ in W7-AS and LHD plotted versus $c / A_{p} t^{2} \equiv\langle\beta\rangle_{\text {eql }}$ as an estimate of an "equilibrium $\beta$ limit" corresponding to $\Delta / a=1 / 2 \quad(\mathrm{c}=2$ for W7-AS, $\mathrm{c}=1$ for LHD). The solid line represents $\langle\beta\rangle=\langle\beta\rangle_{\text {eql }}$ 

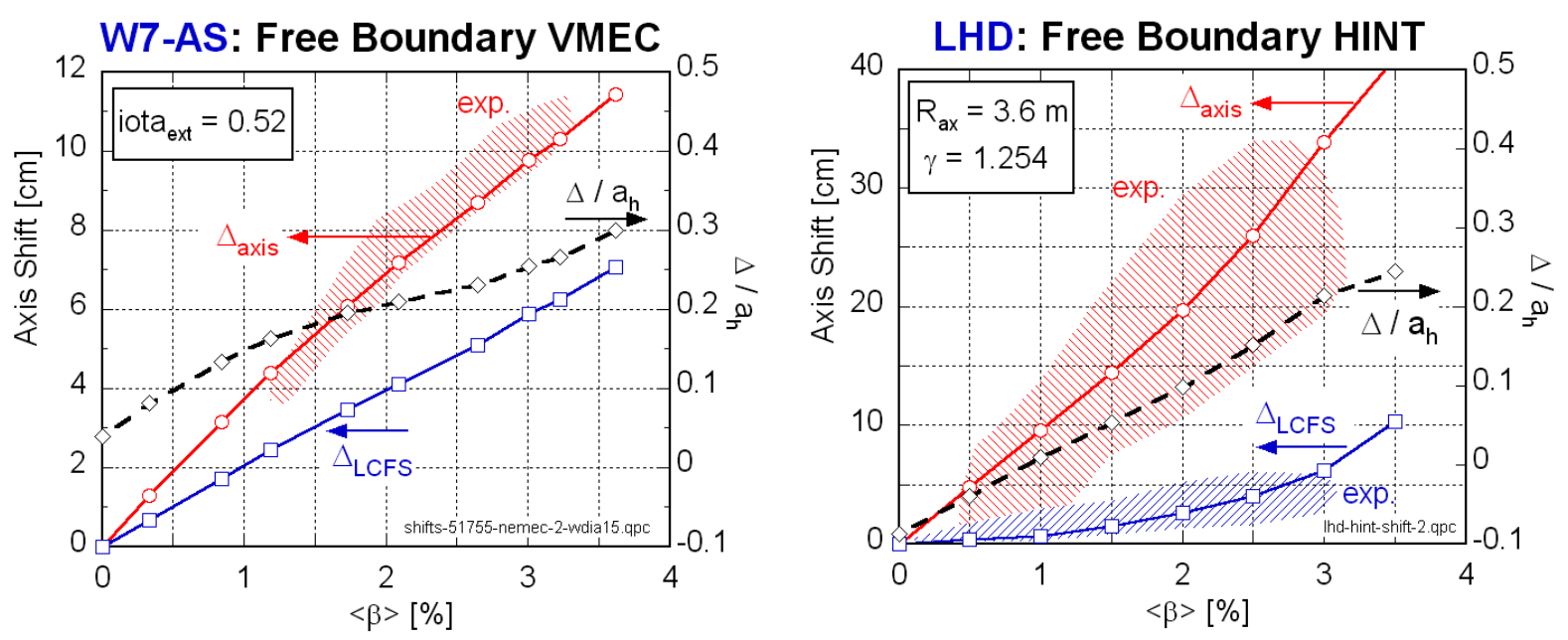

Figure 6. Free boundary VMEC (W7-AS, left) and HINT (LHD, right) predictions of $\beta$ induced horizontal shifts of the plasma axis (red), the LCFS (blue) and their difference (Shafranov shift normalized to horizontal plasma radius $a_{h}$, black dashed line, right scales). The shaded areas represent the range of available experimental data. 

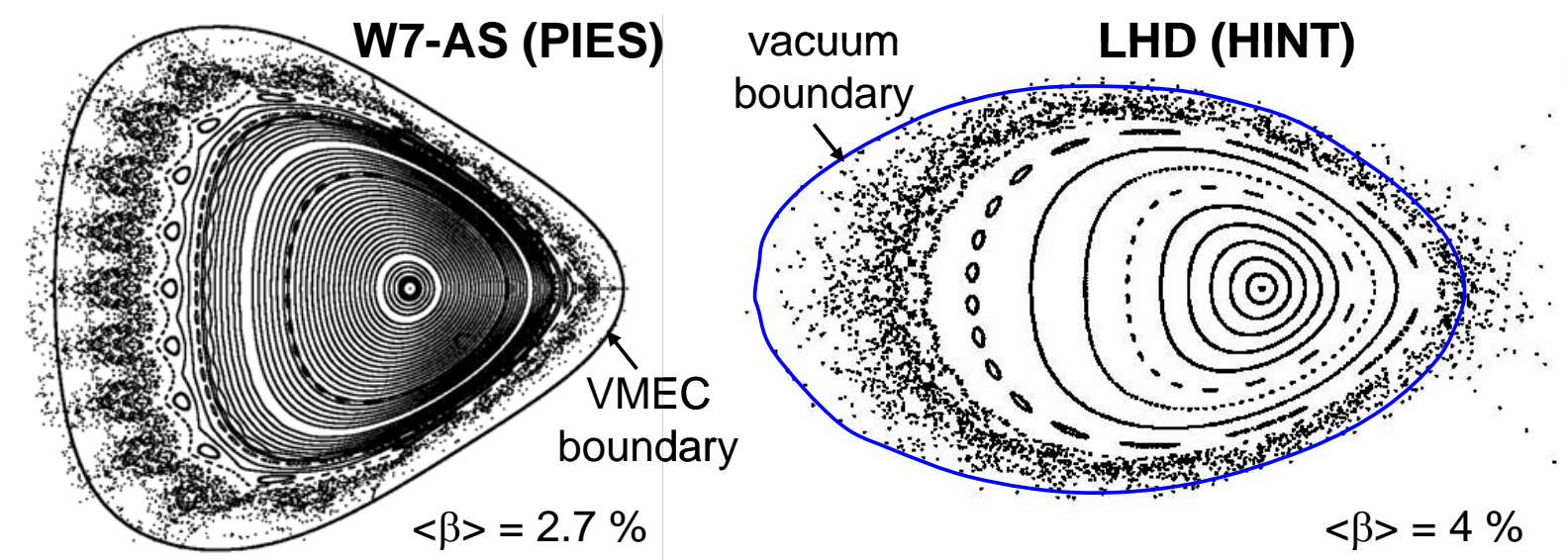

Figure 7. Free boundary high- $\beta$ equilibria as calculated for W7-AS with PIES (left) and for LHD with HINT (right). A large fraction of flux surfaces is predicted to be degraded by island formation and generation of stochastic field regions. 


\section{a) W7-AS: Mercier Stability Diagram}

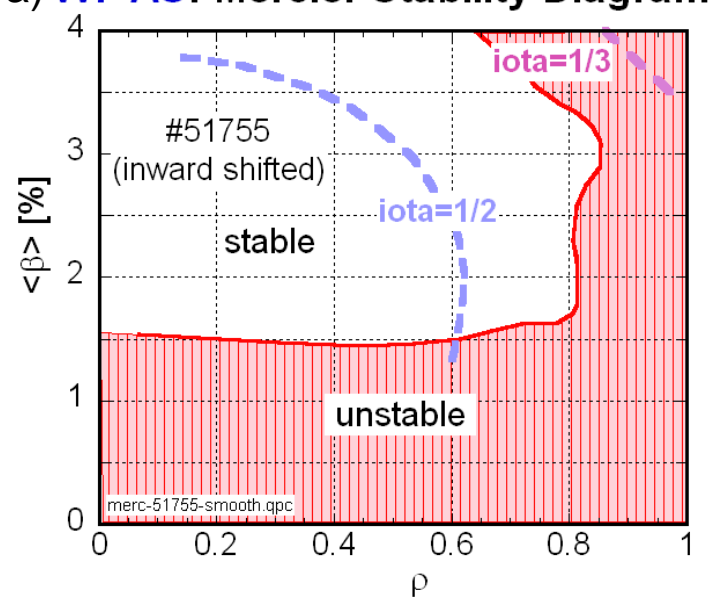

b) LHD: Mercier Stability Diagram

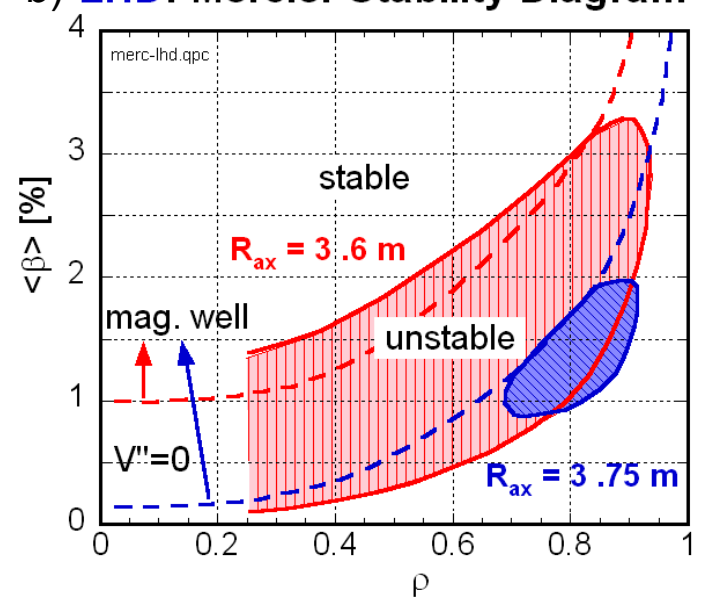

Figure 8. Mercier stability diagrams for W7-AS (left) and LHD (right) configurations. The W7-AS and LHD $\left(R_{a x}=3.6 \mathrm{~m}\right)$ configurations are inward shifted and less stable. 

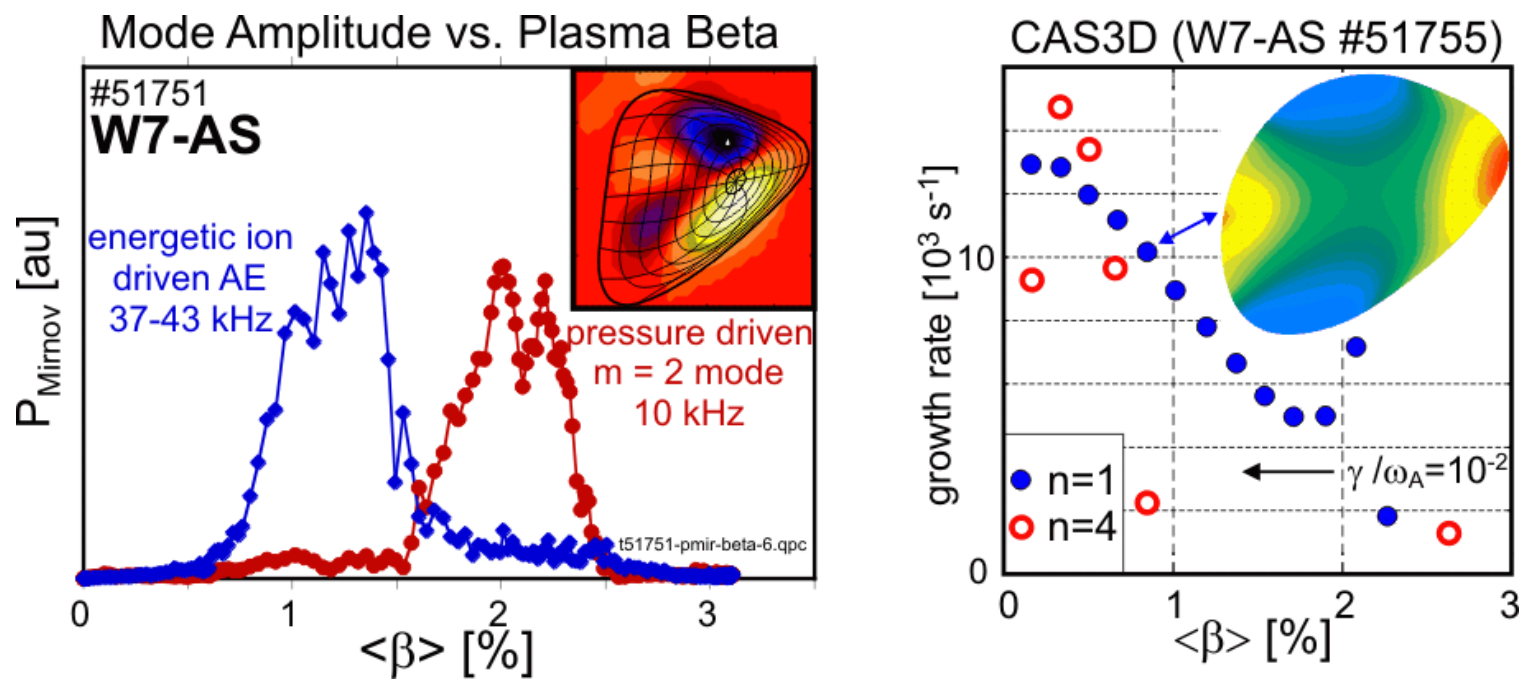

Figure 9. Mode amplitudes in W7-AS depending on $\beta$. The observed $\mathrm{m} / \mathrm{n}=2 / 1$ pressure driven mode (left, inset: X-ray tomogram) dissappears above $\langle\beta\rangle=2.5 \%$ in agreement with vanishing growth rates of global ideal modes as predicted by CAS3D (right). 

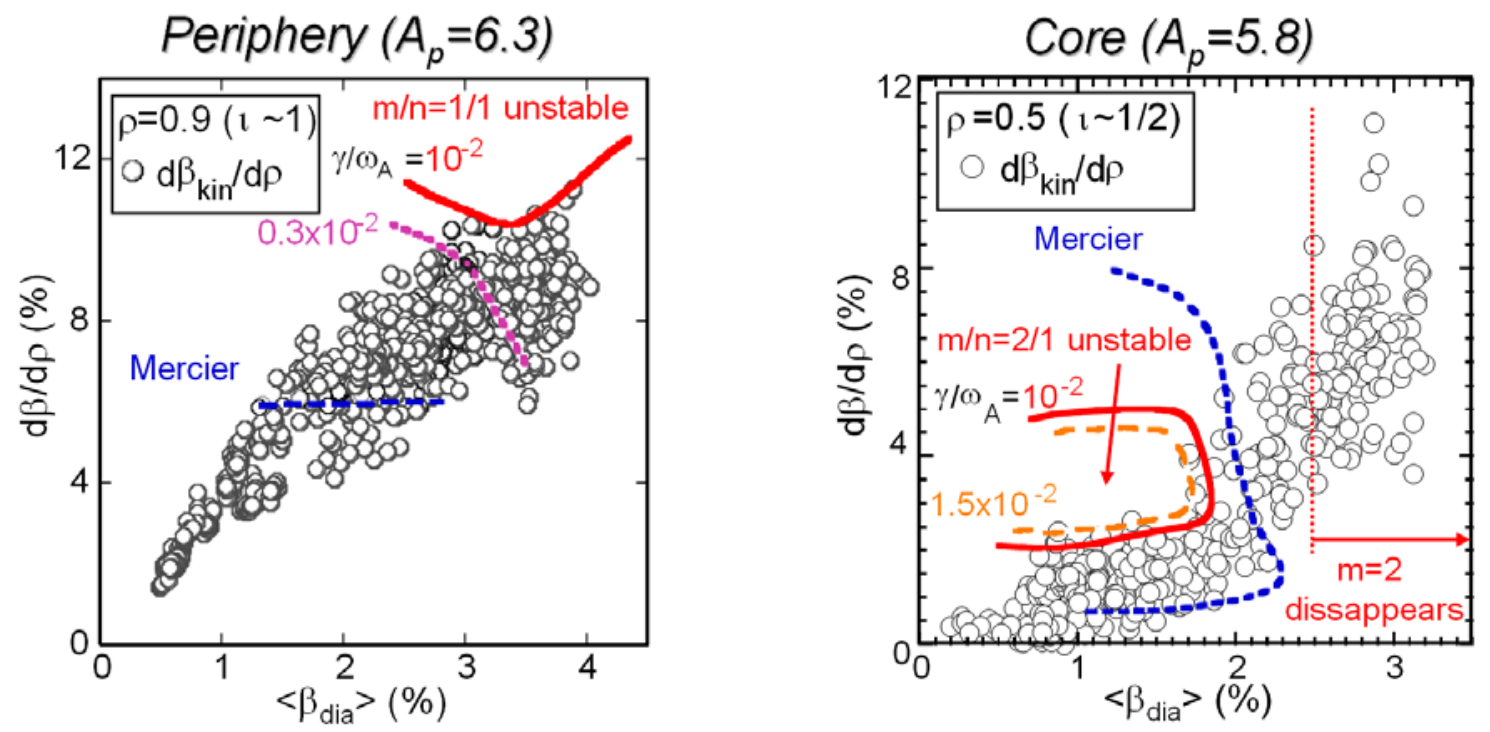

Figure 10. Observed gradients of $\beta$ in LHD at the plasma periphery ( $\rho=0.9$, left) and in the core $(\rho=0.5$, right $)$ plotted against $\langle\beta\rangle$. Stability thresholds for the Mercier stability are significantly violated in experiment. The threshold for low-n ideal interchange modes with growth rates $\gamma / \omega_{A}=10^{-2}$ is not exceeded in experiment. The presence of observed MHD modes correlates with the Mercier unstable region. 

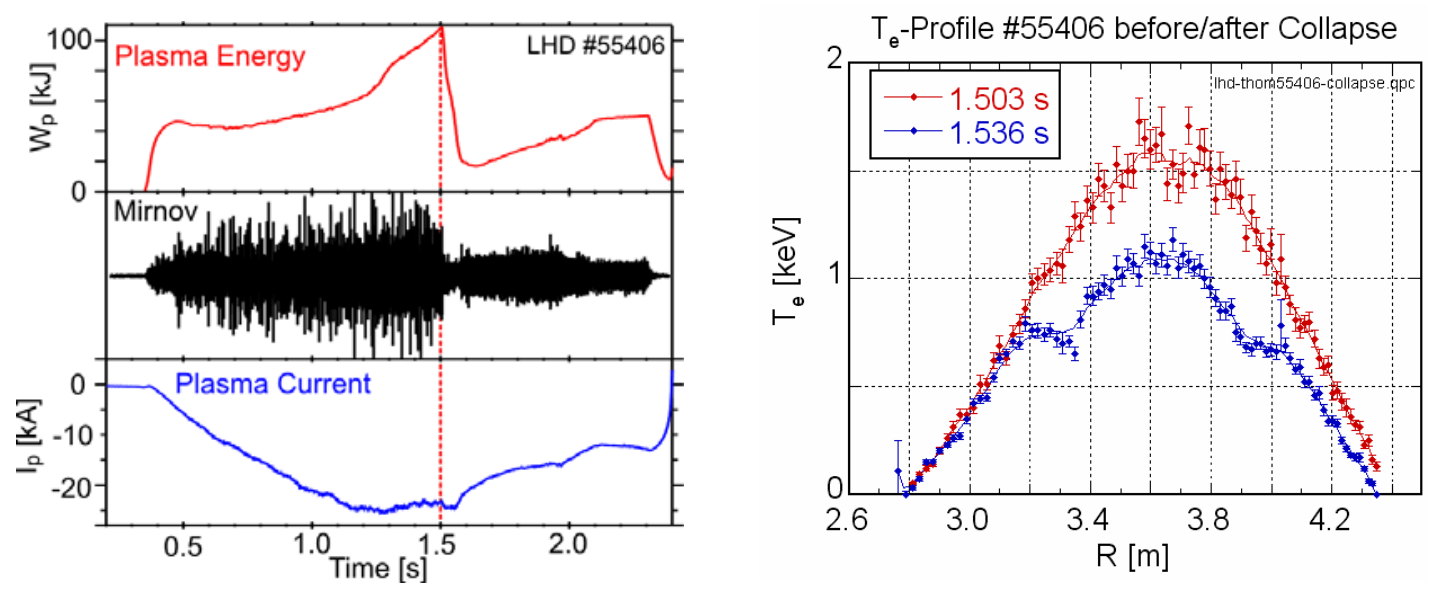

Figure 11. Collapse of plasma energy in LHD due to an $m / n=1 / 1$ interchange mode. A small $\gamma$-parameter $(\gamma=1.129)$ along with co-current generates a low shear iota-profile with $t=1$ in the plasma core. A flattened region in the $T_{e}$-profile is formed during the collapse (right). 


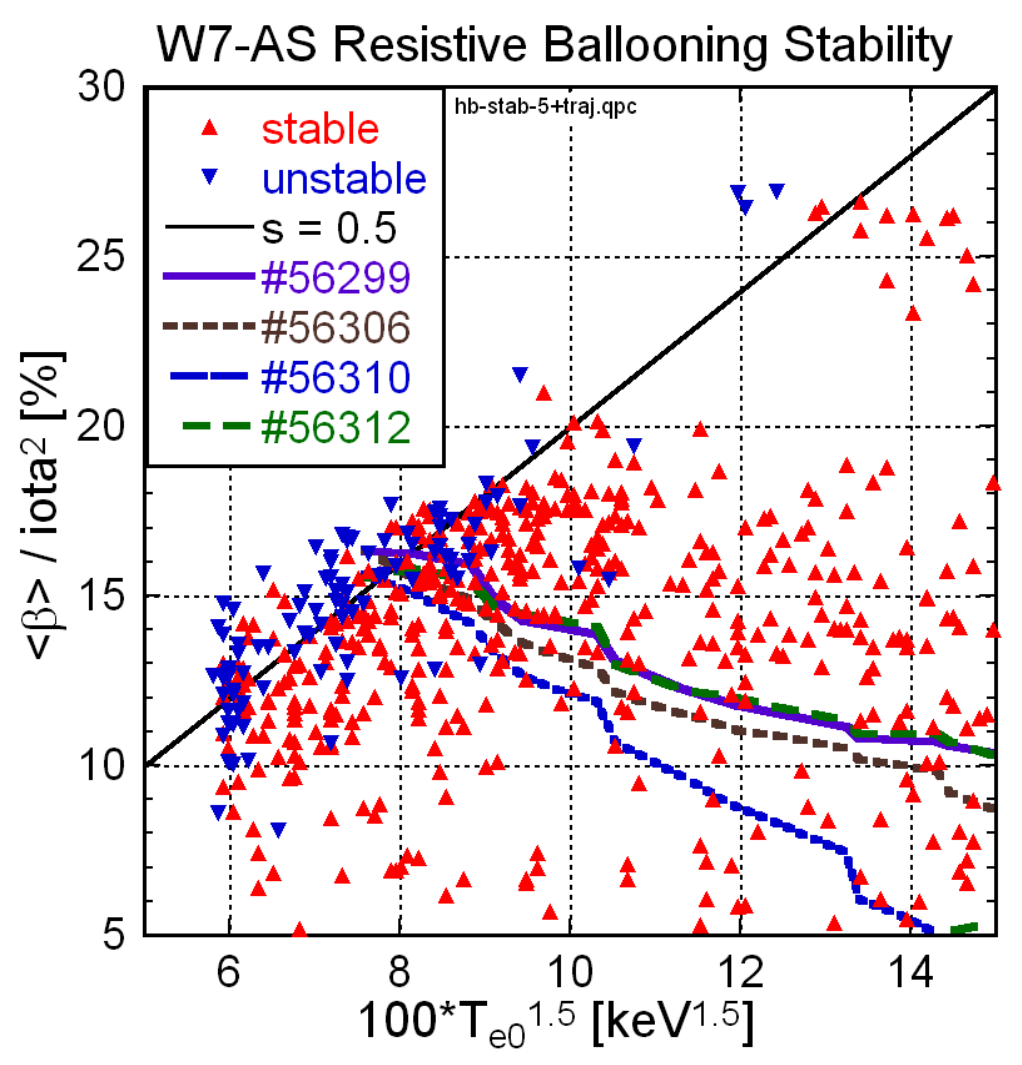

Figure 12. Database of $\left\langle\beta>\right.$ normalized to $t^{2}$ versus $T_{e}^{3 / 2}$ of high- $\beta$ discharges in W7-AS. The parameters just prior to MHD collapses (solid triangles) are close to a critical value of the inverse linear growth rate (dotted line "s $=0.5$ "). The solid lines are the trajectories of individual discharges until the collapse. 